\title{
Integrated Generation and Transmission Expansion Planning Using Generalized Bender's Decomposition Method
}

\author{
Hyoungtae Kim*, Sungwoo Lee* and Wook Kim ${ }^{\dagger}$
}

\begin{abstract}
A novel integrated optimization method based on the Generalized Bender's Decomposition (GBD) is proposed to combine both generation and transmission expansion problems. Most of existing researches on the integrated expansion planning based on the GBD theory incorporate DC power flow model to guarantee the convergence and improve the computation time. Inherently the GBD algorithm based on DC power flow model cannot consider variables and constraints related bus voltages and reactive power. In this paper, an integrated optimization method using the GBD algorithm based on a linearized AC power flow model is proposed to resolve aforementioned drawback. The proposed method has been successfully applied to Garver's six-bus system and the IEEE 30-bus system which are frequently used power systems for transmission expansion planning studies.
\end{abstract}

Keywords: Generation expansion planning, Transmission expansion planning, Integrated optimization, AC power flow model, Generalized Bender's Decomposition

\section{Introduction}

An integrated generation and transmission expansion planning (hereafter IGTEP) is to decide the types and numbers of generators and transmission lines that should be added to the power system, and the appropriate time to add them, so that future electricity demand and the reliability conditions can be met at the least cost. Researches on the optimization of IGTEP started late 1970s, but the early researches were rather confined to small scale or reduced power systems because the computation time and memory usage are intractably increased as the size of the problem increases. Application to the practical scale system has been very rare [1-3]. Therefore, the problem has usually been divided into two separate problems: generation expansion planning and transmission expansion planning.

Recently researches on IGTEP problem started to revive due to mainly two facts: the time and cost to build transmission lines are greatly increased because of NIMBY syndrome against high-voltage transmission lines, and the addressable size of the optimization problems becomes much larger due to the development of the modern computer technology and cheap memory cost. Several researches on optimization of the large scale IGTEP problem have been published recently [4-18].

The optimization of IGTEP problem, by nature, is required to incorporate either $\mathrm{DC}$ or $\mathrm{AC}$ power flow model to calculate the active or reactive power flows through network. Each model has pros and cons in terms of

$\dagger$ Corresponding Author: Dept. of Electrical Engineering, Pusan National University, Korea. (kimwook@pusan.ac.kr)

* Dept. of Electrical Engineering, Pusan National University, Korea. (kimhyoungtae@pusan.ac.kr, ccobungi@naver.com)

Received: February 20, 2015; Accepted: July 7, 2015 convergence and accuracy. While DC model provides faster convergence, it cannot consider the quantities and constraints related to reactive power and bus voltage. And vice versa, AC model can consider various constraints related to reactive power and bus voltage, but it requires long convergence time and sometimes it converges to a poor local optimum due to nonlinearity of $\mathrm{AC}$ power flow model. For this reason, most of the existing researches are based on DC power flow model and only limited number of researches are based on AC power flow model.

The optimization of IGTEP problem inherently formulated as a Mixed Integer Nonlinear Programming (MINLP) due that it contains the binary decision variables and the nonlinear constraints [19]. Not being a rigorous classification, the existing researches on the optimization of IGTEP problem can be categorized into two groups: 1) methods based on combinatorial search such as branch and bound and genetic algorithm [18],2) methods based on the decomposition theory such as Generalized Bender's decomposition $[4,5]$.

The relative strength of the combinatorial search method compared to the decomposition method is its generality. The method can be applied to any type of power systems without any problem-specific restriction and it guarantees asymptotic convergence to the global optimal solution in theory. However, the most significant drawback is that its computation time and memory usage are increased exponentially according to the size of the system. The computation time can significantly be reduced by fathoming, but it becomes intractably long when applied to the large scale real power system. Therefore, the combinatorial search method is only applicable to a relatively small scale system or reduced system. The authors found in the previous research results that the 
largest size of power system to which the combinatorial search method can be applied is the system with less than 20 buses [18].

Meanwhile, the decomposition methods such as Bender's Decomposition (BD), Outer Approximation, Generalized Bender's Decomposition, Generalized Outer Approximation, Generalized Cross Decomposition, etc., are based on the theory that the original problem can be decomposed into two sub-problems under certain regularity assumptions. The methods based on the decomposition theory have the following merits [20]:

1) The given optimization problem can be decomposed into a number of independent problems, each involving different decision variables.

2) The given optimization problem takes a well-known special structure for which efficient algorithms are available.

3) The given optimization problem becomes convex in continuous variables even though it is non-convex in the joint continuous-integer domain.

The IGTEP problem inherently consists of two subproblems, which are: (1) determining the optimal investments in new generation and transmission capacity, and (2) determining the system operating cost (mainly fuel cost of generators) and supply reliability, respectively. Therefore, the application of decomposition methods to the IGTEP problem is very intuitive [2].

Among various decomposition methods, the Generalized Bender's Decomposition (GBD) method is one of the most frequently used ones for IGTEP problem, which guarantees the convergence under specific conditions. Especially, the GBD method has been applied to the transmission expansion planning problem extensively [21-25]. A couple of research results that applied GBD method to the optimization of IGTEP problem are found in the literature $[2,4,5]$.

However, the biggest problem of the GBD method is that it only guarantees the convergence when the subproblem is convex. Hence, most of the existing researches on IGTEP based on GBD method incorporate the (linearized) DC power flow model to guarantee the convergence conditions [1, 21-25].

Thus, the main objective of this paper is to find a general way to apply the GBD method to IGTEP problem with AC power flow model, which guarantees convergence. It is unavoidable to use AC power flow model if we want to consider the constraints related to reactive power and/or bus voltages. The key idea is to use the linearized version of AC power flow model to guarantee the convergence conditions of GBD theory. We found that the proposed method improves not only the convergence itself but also the convergence speed of the overall optimization procedure.

In the following section the mathematical formulation of the proposed method is described. The proposed method is applied to the Garver's six-bus power system and the IEEE 30-bus system which are frequently used sample power systems for the transmission expansion planning researches. The simulation results are shown in Section 3. Conclusions are given in the last section.

\section{Mathematical Formulation}

\subsection{Nomenclature}

$\mathrm{i}, \mathrm{j} \quad$ Index of bus

$\mathrm{g}$ Index of generator

id Index of $\mathrm{T} / \mathrm{L}$ circuits between $\mathrm{i}$ and $\mathrm{j}$

$\Omega_{g} \quad$ Set of all generators including existing generators and new generators

$\Omega_{b} \quad$ Set of all buses including existing buses and new buses

$P G_{g} \quad$ Real power output of generator(MW)

$Q G_{g} \quad$ Reactive power output of generator (MVar)

$P_{i j, i d, t} \quad$ Real power flow between i and j(MW)

$Q_{i j, i d, t} \quad$ Reactive power flow between i and j(MVar)

$\Delta V_{i, t} \quad$ Bus voltage magnitude deviation in pu at bus $i$ at time $\mathrm{t}$

$\Delta V_{i, \max }$ Upper bound on the voltage magnitude deviation in pu at bus $i$

$\Delta V_{i, \min }$ Lower bound on the voltage magnitude deviation in pu at bus $i$

$\theta_{i j, t} \quad$ Phase angle difference between $\mathrm{i}$ and $\mathrm{i}$ at $\mathrm{t}(\mathrm{rad})$

$g_{i j, i d} \quad$ Conductance of existing and prospective $\mathrm{T} / \mathrm{L}$ between $i$ and i(p.u)

$b_{i j, i d} \quad$ Susceptance of existing and prospective $\mathrm{T} / \mathrm{L}$ between $i$ and $j(p . u)$

$P D_{i,} \quad$ Real power demand at bus $\mathrm{i}$ at time $\mathrm{t}(\mathrm{MW})$

$Q D_{i, t} \quad$ Reactive power demand at bus i at $\mathrm{t}$ (MVar)

$a_{g} \quad$ Coefficient of second order term of operation cost function of each generator $\left(\$ / \mathrm{MW}^{2}\right)$

$b_{g} \quad$ Coefficient of first order term of operation cost function of each generator $(\$ / M W)$

$c_{g} \quad$ Constant coefficient of operation cost function of each generator $(\$)$

$C P_{g} \quad$ Investment cost of generators(M\$)

$T C_{i j}^{g}$ Investment cost of $\mathrm{T} / \mathrm{L}$ between $\mathrm{i}$ and $\mathrm{j}(\mathrm{M} \$)$

$d r \quad$ Discount rate

$S_{g, \max }$ Maximum apparent power output of each generator for existing and prospective generators (MVA)

$S_{i j, i d, \text { max }}$ Maximum apparent flow limit on T/L between bus i and j(MVA)

$o_{g, t} \quad$ Binary variable for operation of each generator

$u_{i j, i d, t}$ Binary decision variable for an existing and prospective line between bus $i$ and $j$ at time $t$.

$z_{g, t} \quad$ Binary decision variable for an existing and prospective generator at time $t$. 

$u c_{i j, i d, t}$ Binary decision variable included construction period for a prospective line between buses $i$ and $\mathrm{j}$ at time t.
$z c_{g, t}$ Binary decision variable included construction period for a prospective generator at time $t$.
$L_{g, i} \quad$ Binary variable if generator $\mathrm{g}$ is connected to bus i
$\mathrm{t}, \mathrm{T} \quad$ Index of time and planning period

\subsection{Description of the Generalized Benders' Decom- position (GBD) method}

The GBD method proposed by Geoffrion is a generalized version of the Bender's Decomposition (BD), which is an optimization theory based on the nonlinear convex duality to deal with nonlinear programming problem such as MINLP [26]. It guarantees that the optimal solution is found in a finite number of iterations under certain convexity and regularity assumptions $[4,5,20,26]$. It is assumed that the original optimization problem is given as follows:

$$
\begin{gathered}
\min _{x, y} f(x, y) \\
\text { s.t } h(x, y)=\alpha \\
g(x, y) \leq \beta \\
x \in X \subseteq R^{n} \\
y \in Y=\{0,1\}^{q}
\end{gathered}
$$

where $x$ and $y$ are vectors for operational variables and investment decision variables, respectively.

The objective function in Eq. (1) can be rewritten separately for $y$ and $x$ using 'min' and 'inf' operators as follows:

$$
\begin{gathered}
\min _{y} \inf _{x} f(x, y) \\
\text { s.t } h(x, y)=\alpha \\
g(x, y) \leq \beta \\
x \in X \subseteq R^{n} \\
y \in Y=\left\{0,1^{q}\right.
\end{gathered}
$$

If we define $v(y)$ as $v(y)=\inf _{x} f(x, y)$ then Eq. (2) can be expressed as follows:

$$
\begin{gathered}
\min _{y} v(y) \\
\text { s.t } \quad y \in Y \cap V
\end{gathered}
$$

where, the set $V$ is defined as $V=\{y \mid h(x, y)=\alpha, g(x, y)$ $\leq \beta$ for some $x \in X\}$. In the above equation, $v(y)$ and $V$ can be described as dual representations by "Geoffrion's theorem related to the dual representation" as follows [20, 26]:

Dual representation of $V$ :

$$
0 \geq \inf \bar{L}(x, y, \bar{\lambda}, \bar{\mu}), \quad \forall \bar{\lambda}, \bar{\mu} \in \Lambda
$$

where, $\Lambda=\left\{\bar{\lambda} \in R^{m}, \bar{\mu} \in R^{p}: \bar{\mu} \geq 0, \sum_{i=1}^{p} \bar{\mu}=1\right\}$

\section{Dual representation of $v(y)$ :}

$$
v(y)=\inf _{x \in X} \sup _{\lambda, \mu \geq 0} L(x, y, \lambda, \mu), \quad \forall y \in Y \cap V
$$

where,

$$
\begin{aligned}
& L(x, y, \lambda, \mu)=f(x, y)+\lambda(h(x, y)-\alpha)+\mu(g(x, y)-\beta) \\
& L(x, y, \bar{\lambda}, \bar{\mu})=\bar{\lambda}(h(x, y)-\alpha)+\bar{\mu}(g(x, y)-\beta) \\
& \lambda, \mu: \text { Dual multiplier of feasible primal problem } \\
& \bar{\lambda}, \bar{\mu}: \text { Dual multiplier of infeasible primal problem }
\end{aligned}
$$

The dual representation described as Eq. (5) can further be decomposed into two sub-problems dubbed as "master problem" and "primal problem", respectively. These two problems form a saddle point problem where the minimum of the master problem and the maximum of the primal problem intersect each other.

The basic idea of the GBD method is that it utilizes the concept of "complicating variable" in order to find the optimal solution of the problem given by Eq. (5) which is equivalent to Eq. (1) [20]. The original problem described as Eq. (3) or Eq. (5) contains the instrument variables $x$ and $\mu$. But the master problem or the 'sup' problem only considers $\mu$ as the instrument variables and $x$ is considered as the input parameters (decided by the primal problem or the 'inf' problem), whereas the primal problem or the 'inf' problem considers $x$ as the instrument variables and $\mu$ as the input parameters (decided by the master or the 'sup' problem). These two 'sup' problem and 'inf' problem should be solved alternately until the values of objective functions of two problems converge to each other as shown in Fig. 1.

The difference between the values of the objective functions of the master problem and the primal problem is called "duality gap". During the initial phase, the duality gap generally may not be small, but gradually converges to

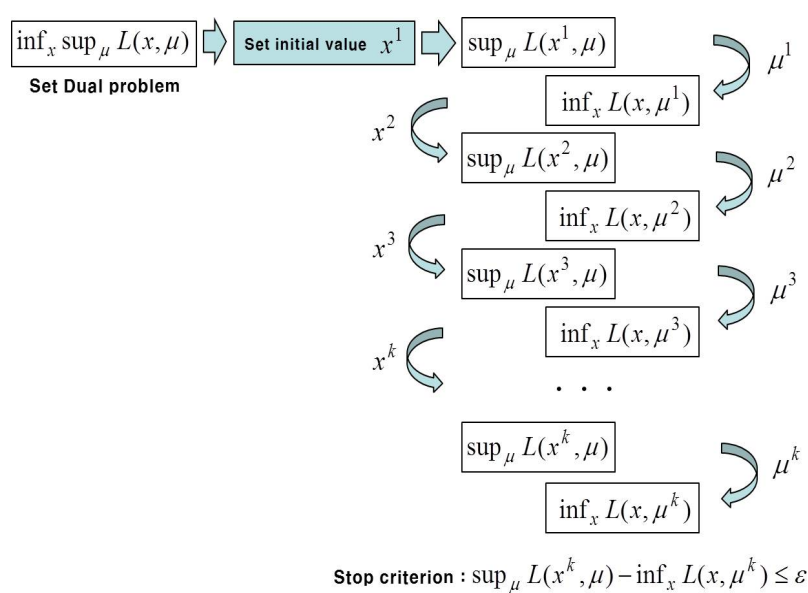

Fig. 1. Concept of Decomposition Method 
small value in a certain number of iterations. When the duality gap is converged to zero, the solutions of the master and primal problems are same and hence the value represents the optimal solution of the original problem. However, the convergence can only be guaranteed when the following conditions are met $[20,26]$ :

C1. $Y$ be a nonempty subset of $V$. where $V=\{y: h(x, y)=\alpha, g(x, y) \leq \beta\}$ for some $x \in X$

C2. $X$ be a nonempty convex set.

C3. $f, g$ be convex on $X$ for each fixed $y \in Y$.

C4. $h$ be linear on $X$ for each fixed $y \in Y$.

C5. $f, g, h$ be continuous on $X \times Y$.

C6. The set of optimal multiplier vectors for the primal problem be nonempty for all $y \in Y$, and uniformly bounded in some neighborhood of each such point.

Using the definition of supremum and the assumption that $v(y)$ is bounded all $y \in Y \cap V$, the master problem can be stated as the following equation:

$$
\begin{gathered}
\min _{y \in Y, \mu_{B}} \mu_{B} \\
\text { s.t } \mu_{B} \geq \min _{x \in X} L(x, y, \lambda, \mu), \quad \forall \lambda, \forall \mu \geq 0 \\
0 \geq \min _{x \in X} \bar{L}(x, y, \bar{\lambda}, \bar{\mu}), \quad \forall(\bar{\lambda}, \bar{\mu}) \in \Lambda
\end{gathered}
$$

And the primal problem, which is related to minimization of the operation cost for fixed investment decision variables, can be stated as follows:

$$
\begin{gathered}
\min _{x} f\left(x, y^{k}\right) \\
\text { s.t } \quad h\left(x, y^{k}\right)=\alpha \\
g\left(x, y^{k}\right) \leq \beta \\
x \in X \subseteq R^{n}
\end{gathered}
$$

where, $\mathrm{k}$ is the iteration number. The optimal solution $x$ for fixed $y(y \in Y \cap V)$ and multipliers are decided by solving the above primal problem. Next, the solution procedure of the GBD method can be summarized as follows:

Step 1: Let an initial point $y^{1} \in Y \cap V$. Solve the resulting primal problem $P\left(y^{1}\right)$ and obtain primal solution $x^{1}$ and optimal multipliers; vectors $\lambda^{1}, \mu^{1}$. Set the counters $\mathrm{k}=1$ for feasible and $\mathrm{l}=1$ for infeasible and the current upper bound UBD $=v\left(y^{1}\right)$. Select the convergence tolerance $\varepsilon \geq 0$.

Step 2: Solve the master problem. If $\mathrm{UBD}-\mathrm{LBD} \leq \varepsilon$, then terminate.

Step 3(a): (Feasible primal problem) The primal has $v(\hat{y})$ finite with an optimal solution $\hat{x}$ and optimal multiplier vectors $\hat{\lambda}, \hat{\mu}$. Update the upper bound $\mathrm{UBD}=\min \{\mathrm{UBD}, v(\hat{y})\}$. If UBD-LBD $\leq \varepsilon$, then terminate. Otherwise, set. $\mathrm{k}=\mathrm{k}+1, \lambda^{k}=\hat{\lambda}$ and $\mu^{k}=\hat{\mu}$. Return to step. 2.

Step 3(b): (Infeasible primal problem) The primal does not have a feasible solution for $y=\hat{y}$. Solve a feasibility problem (e.g., the $1_{1}$-minimization) to determine the multiplier vectors $\overline{\hat{\lambda}}, \overline{\hat{\mu}}_{-}$of the feasibility problem. Set $\mathrm{l}=1+1, \bar{\lambda}^{l}=\overline{\hat{\lambda}}$, and $\bar{\mu}^{l}=\overline{\hat{\mu}}$. Return to step. 2 .

\subsection{Application of GBD method to the integrated optimization of generation and transmission expansion planning problem}

The optimization of IGTEP problem generally consists of two sub-problems: an investment cost minimization which is generally formulated as the mixed integer programming (MIP) and the economic dispatch problem which is generally formulated as the nonlinear programing (NP). Naturally, the IGTEP problem has a mathematical structure to which the GBD method can easily be applied. Therefore, the GBD method is extensively applied to the power system planning problem such as the transmission expansion planning since early 1980s [21-25].

However, one of the obstacles to applying the GBD method to IGTEP problem is that the general mathematical formulation of IGTEP problem does not satisfy the convergence conditions $\mathrm{C} 1 \sim \mathrm{C} 6$ described above mainly due to the nonlinearity of the power flow equation. In order to overcome this problem, most of the existing researches are based on the linearized DC power flow [21-24]. Some researches based on the nonlinear $\mathrm{AC}$ power flow equation have been published, but they are lacking of generality because they cannot guarantee the convergence of the method $[4,5,25]$.

Hence, this paper suggests a new way of applying the GBD method to the IGTEP problem which guarantees the convergence conditions $\mathrm{C} 1$ through $\mathrm{C} 6$ by linearization of the various constraints without significant damage to the accuracy of the original problem.

In order to find the optimal solution of the IGTEP using GBD method successfully, it is obvious that some of the constraints should be modified or linearized because GBD method based on full AC power flow model (Eq.(18) and (19) below) does not guarantee the convergence conditions C1 C6. In addition, the linearized AC power flow model can significantly reduce the computation time and the convergence. So, in this paper the simplifications are performed based on the following assumptions:

A1. Technical characteristic of generators, such as rampup/down rates and minimum up/down times, etc., are ignored.

A2. When the investment costs of generator and transmission lines are calculated, the real power loss is ignored because resistance of the transmission lines is usually significantly smaller than line reactance.

A3. The operation cost of transmission lines and the 
salvage values of the generators and transmission lines at the end of the planning period are ignored. Also, the start - up cost of the each generator is ignored.

A4. Characteristics of newly built transmission lines between bus $i$ and $j$ are identical with existing transmission lines between bus $i$ and $j$.

A5. All impracticable candidates of transmission lines based on topology analysis are excluded from the planning model.

The above assumptions are commonly accepted in the previous long-term generation and transmission expansion planning studies although not having been without controversies [19].

\subsection{Mathematical formulation}

\subsubsection{Objective function}

The objective function of the IGTEP problem is the sum of the operation and investment (or construction) costs of generation and transmission systems, which can be formulated as follows:

$$
f(x, y)=\sum_{t}^{T}(\text { Operation } \operatorname{cost}(\mathrm{OC})+\text { Investment } \operatorname{cost}(\mathrm{IC}))
$$

where, OC : $\sum_{g}^{\Omega_{g}} \frac{\left(c_{g} o_{g, t}+b_{g} P G_{g, t}+a_{g} P G_{g, t}{ }^{2}\right)}{(1+d r)^{t}}$

$$
\mathrm{IC}: \frac{\sum_{\substack{i, j, i d \\ i \neq j}}^{\Omega_{b}}\left(u c_{i j, i d, t}-u c_{i j, i d, t-1}\right) T C_{i j, i d}+\sum_{g}^{\Omega_{g}}\left(z c_{g, t}-z c_{g, t-1}\right) C P_{g}}{(1+d r)^{t-1}}
$$

Here, we ignore the operation cost of the transmission lines without loss of generality because it is much smaller than that of generators.

\subsubsection{Constraints}

The following inequality and equality constraints are considered for the optimization of the IGTEP problem.

\section{Limits on the apparent power of the generators:}

In general the apparent power output of a generator is characterized as a nonlinear inequality constraint and the real and reactive power output characteristic for minimum output and maximum output of a generator are considered as the following equations:

$$
\begin{gathered}
P G_{g, t}{ }^{2}+Q G_{g, t}{ }^{2} \leq S_{g, \text { max }}{ }^{2}, \forall t, \forall g \in \Omega_{g} \\
P G_{g, t} \leq z_{g, t} P_{g, \text { max }}, \quad \forall t, \forall g \in \Omega_{g}
\end{gathered}
$$

$$
\begin{gathered}
Q G_{g, t} \leq z_{g, t} Q_{g, \text { max }}, \quad \forall t, \forall g \in \Omega_{g} \\
o_{g, t} P_{g, \text { min }} \leq P G_{g, t} \leq o_{g, t} P_{g, \text { max }}, \quad \forall t, \forall g \in \Omega_{g} \\
o_{g, t} Q_{g, \text { min }} \leq Q G_{g, t} \leq o_{g, t} Q_{g, \text { max }}, \quad \forall t, \forall g \in \Omega_{g}
\end{gathered}
$$

Eq. (9) is a constraint to limit apparent power output of each generator. Eq. (10) and (11) are constraints to determine binary variables for the prospective generators. Eq. (12) and (13) are constraints related to output characteristic of each generator and to determine binary variables for operation of existing and newly built generators.

Though Eq. (9) satisfies the convergence conditions C1 through C6, but it makes the convergence very slow due to its severe nonlinearity. Eq. (9) can be approximated by the following four inequalities, which are quite close to the original nonlinear loading capability curve as shown in Fig. $2[18,27]$ :

$$
\begin{aligned}
& Q G_{g, t} \leq a_{1} P G_{g, t}+a_{2} S_{g, \max }, \forall t, \forall g \in \Omega_{g} \\
& Q G_{g, t} \leq b_{1} P G_{g, t}+b_{2} S_{g, \max }, \forall t, \forall g \in \Omega_{g} \\
& Q G_{g, t} \geq c_{1} P G_{g, t}+c_{2} S_{g, \max }, \quad \forall t, \forall g \in \Omega_{g} \\
& Q G_{g, t} \geq d_{1} P G_{g, t}+d_{2} S_{g, \max }, \quad \forall t, \quad \forall g \in \Omega_{g}
\end{aligned}
$$

where $a_{i}$ and $b_{i}$ are all constants.

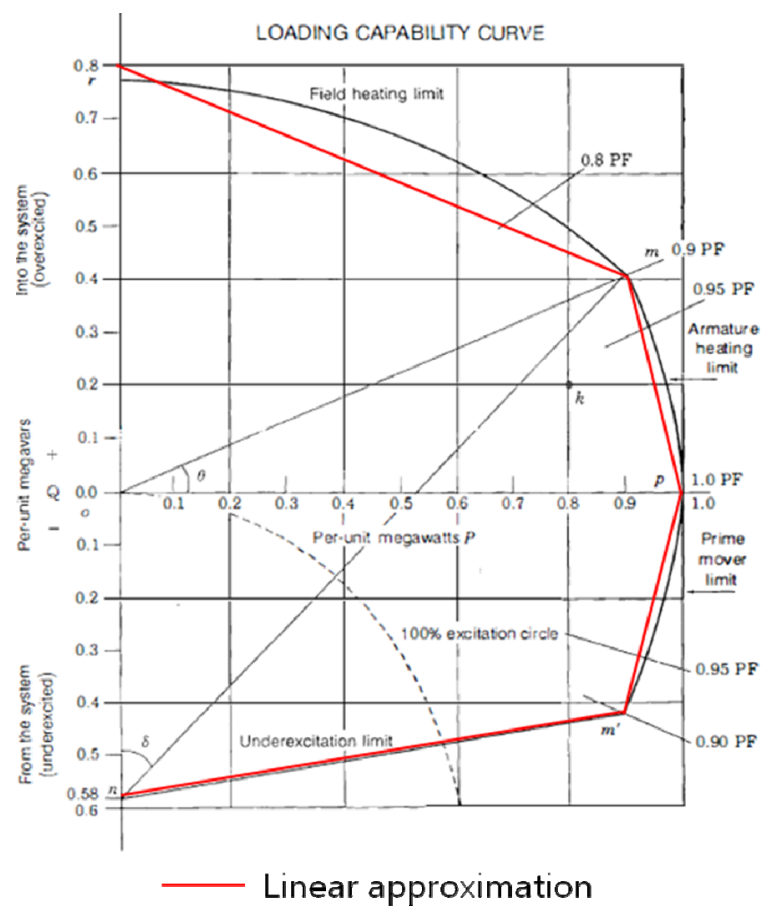

Fig. 2. Linearization of loading capability curve [27]

AC Power Flow:

The general nonlinear AC power flow is calculated as the following equations: 


$$
\begin{aligned}
& P_{i j, i d, t}=V_{i, t}{ }^{2} g_{i j, i d}-V_{i, t} V_{j, t}\left(g_{i j, i d} \cos \theta_{i j, t}+b_{i j, i d} \sin \theta_{i j, t}\right) \\
& Q_{i j, i d, t}=-V_{i, t}{ }^{2} b_{i j, i d}-V_{i, t} V_{j, t}\left(g_{i j, i d} \sin \theta_{i j, t}-b_{i j, i d} \cos \theta_{i j, t}\right)
\end{aligned}
$$

Based on the assumption A2 which allows us to neglect resistance of the transmission line, Eq. (18) and (19) can be approximated as the followings:

$$
\begin{gathered}
P_{i j, i d, t}=-V_{i, t} V_{j, t} b_{i j, i d} \sin \theta_{i j, t} \\
Q_{i j, i d, t}=-V_{i, t}{ }^{2} b_{i j, i d}+V_{i, t} V_{j, t} b_{i j, i d} \cos \theta_{i j, t}
\end{gathered}
$$

Notice that Eq.(20) and (21) still do not satisfy the convergence properties $\mathrm{C} 1 \sim \mathrm{C} 6$ due to their nonlinearities. Therefore, the above equations are to be further linearized using the following additional assumptions [28].

1) All the bus voltage magnitudes are close to 1.0 per unit.

2) The angle difference across a line is small so that $\sin \theta \approx \theta$ and $\cos \theta \approx 1$ can be applied. This assumption is valid at the transmission level where the active power flow dominates the apparent power flow in the lines.

By the above assumptions, the bus voltage magnitude can be written as $V_{i, t}=1+\Delta V_{i, t}$ where $\Delta V_{i, t}$ is expected to be small. Substituting $1+\Delta V_{i, t}$ into Eq. (20) and (21), and neglecting higher order terms, the Eq. (20) and (21) can be approximated as follows, which satisfy the convergence conditions C1 through C6 [28]:

$$
\begin{gathered}
P_{i j, i d, t}=-\left(1+\Delta V_{i, t}+\Delta V_{j, t}\right) u_{i j, t} b_{i j, i d} \theta_{i j, t} \\
Q_{i j, i d, t}=-\left(\Delta V_{i, t}-\Delta V_{j, t}\right) u_{i j, i d . t} b_{i j, i d} \\
\text { for } \forall t, \forall i, j \in \Omega_{b}, \quad \forall i \neq j .
\end{gathered}
$$

$\underline{\text { Real and reactive power transmission flow limit: }}$

The constraints on the real and reactive power transmission flow can be modeled by the following nonlinear inequality:

$$
P_{i j, i d, t}{ }^{2}+Q_{i j, i d, t}{ }^{2} \leq u_{i d, i d, t} S_{i j, i d, \max }{ }^{2}, \forall t, \forall i, j, i d \in \Omega_{b}
$$

Similar to the above case, this constraint needs to be linearized to improve the convergence speed. Hence, it is also non-convex and hence to be linearized as follows:

$$
\begin{aligned}
Q_{i j, i d, t} & \leq \alpha P_{i j, i d, t}+u_{i j, t} S_{i j, i d, \max }\left(\sin \theta_{k}-\alpha \cos \theta_{k}\right), \\
\alpha & =\frac{\sin \theta_{k}-\sin _{k+1}}{\cos \theta_{k}-\cos \theta_{k+1}}, k=1,2, \cdots, n-1
\end{aligned}
$$

for $\forall t, \forall i, j, i d \in \Omega_{b}$. The above inequality is obtained by linearizing the Eq. (24) as shown in Fig. 3. It is obvious that the result become more exact if $n$ is set to the larger

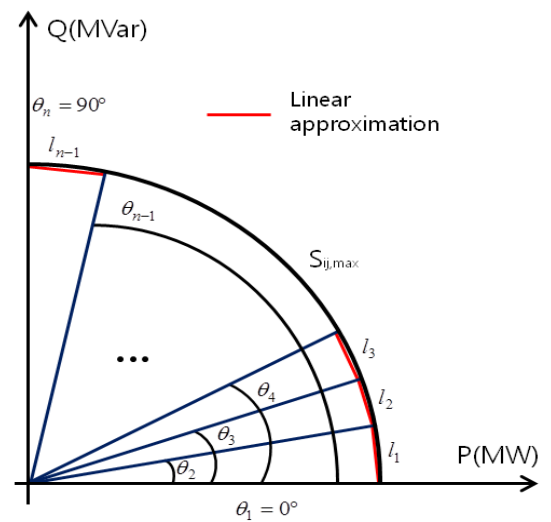

Fig. 3. Linear approximation for transmission flow limit

number, we found that the result is acceptable even if $n$ is set to the smallest value or 2 [18].

\section{Node balance (Kirchhoff's $1^{\text {st }}$ Law):}

The node balance equations (the Kirchhoff's first law for real and reactive powers) at each node are modeled as follows:

$$
\begin{aligned}
& \sum_{g}^{\Omega_{g}} P G_{g, t} L_{g, i}-P D_{i, t}=\sum_{j, i d}^{\Omega_{b}} P_{i j, i d, t}, \quad \forall t, i \in \Omega_{b} \\
& \sum_{g}^{\Omega_{g}} Q G_{g, t} L_{g, i}-Q D_{i, t}=\sum_{j, i d}^{\Omega_{b}} Q_{i j, i d t}, \quad \forall t, i \in \Omega_{b}
\end{aligned}
$$

Demand and supply conditions for real and reactive power:

$$
\begin{aligned}
& \sum_{g}^{\Omega_{g}} P G_{g, t}=\sum_{i}^{\Omega_{b}} P D_{i, t}+\sum_{i, j, i d}^{\Omega_{b}} 0.5 P l_{i j, i d, t}, \quad \forall t \\
& \sum_{g}^{\Omega_{g}} Q G_{g, t}=\sum_{i}^{\Omega_{i}} Q D_{i, t}+\sum_{i, j, i d}^{\Omega_{b}} 0.5 Q l_{i j, i d, t}, \quad \forall t
\end{aligned}
$$

Eqs. (28) and (29) are the demand and supply conditions for active and reactive powers, respectively. Also, the real power loss $P l$ and reactive power loss $Q l$ are multiplied by 0.5 as in Eq. (28) and (29) in order to avoid the double calculation of real and reactive power losses.

By the assumption A2, the real and reactive losses on transmission lines are to be ignored and, hence, Eq.(28) and (29) can be modified as follows:

$$
\begin{aligned}
& \sum_{g}^{\Omega_{g}} P G_{g, t}=\sum_{i}^{\Omega_{b}} P D_{i, t}, \quad \forall t \\
& \sum_{g}^{\Omega_{g}} Q G_{g, t}=\sum_{i}^{\Omega_{i}} Q D_{i, t}, \quad \forall t
\end{aligned}
$$

Reserve rate and capacity factor limit: 


$$
\begin{aligned}
\sum_{i}^{\Omega_{b}} P D_{i, t} \leq & \sum_{g}^{\Omega_{g}} z_{g, t} P \max _{g} \leq\left(1+r_{t}\right) \sum_{i}^{\Omega_{b}} P D_{i, t}, \forall t \\
& \frac{P_{g, t}}{P \max _{g}} \leq \rho_{g}, \forall g \in \Omega_{g}, \forall t
\end{aligned}
$$

where, $r_{t}$ : reserve rate at $\mathrm{t}$

$$
\rho_{g} \text { : capacity factor of each generator } g
$$

Eqs. (32) and (33) represent the inequality constraints on the reserve rate and capacity factor during the planning period, respectively.

$\underline{\text { Time to market constraint: }}$

$$
\begin{gathered}
z_{g, t}-z_{g, t-1} \leq \text { GenEntry }_{g, t}, \forall g \in \Omega_{g}, t \\
u_{i j, i d, t}-u_{i j, i d, t-1} \leq \text { TransEntry }_{i j, i d, t}, \forall i, i d \in \Omega_{b}, \forall t
\end{gathered}
$$

where,

GenEntry $_{g, t}$ : parameters for the availability of the newly built generators

TransEntry $_{i j, i d, t}$ : parameters for the availability of the newly built transmission lines (1: available, 0 : unavailable)

Max. number of facilities which can be built in a year:

$$
\begin{gathered}
\sum_{g}^{\Omega_{g, \text { new }}} z_{g, t}-z_{g, t-1} \leq n_{\text {gen }}, \forall t \\
\sum_{i, j, i d}^{\Omega_{b, \text { new }}} u_{i j, i d, t}-u_{i j, i d, t-1} \leq n_{\text {line }}, \forall t \\
\sum_{g}^{\Omega_{g, \text { new }}} z_{g, \text { tlast }} \leq n_{g, \text { total }} \\
\sum_{i, j, \text { id }}^{\Omega_{b, \text { new }}} u_{i j, \text { id }, \text { tlast }} \leq n_{\text {line }, \text { total }}
\end{gathered}
$$

where, $n_{\text {gen }}:$ max. number of new generators (one year) $n_{\text {line }}:$ max. number of new transmission lines (one year)

$n_{g, \text { total }}:$ total number of new generators (total)

$n_{\text {line,total }}:$ total number of new transmission lines (total)

Eqs. (36) and (37) are the constraints on the maximum number of new generators and transmission lines which can be built in a year, respectively. Eqs. (38) and (39) are the constraints on the total number of new generators and transmission lines during the total planning period, respectively.

Other constraints:

$$
\Delta V_{\min } \leq \Delta V_{i, t} \leq \Delta V_{\max }, \forall t, \forall i \in \Omega_{b}
$$

$$
\begin{gathered}
\theta_{i j, \min } \leq \theta_{i j, t} \leq \theta_{i j, \max }, \quad \forall t, \forall i, j \in \Omega_{b} \\
u_{i j, i d, t-1} \leq u_{i j, i d, t}, \quad \forall t, \forall i, j, i d \in \Omega_{b} \\
z_{g, t-1} \leq z_{g, t}, \quad \forall t, \forall g \in \Omega_{g} \\
u c_{i j, i d, t-\text { period }}=u_{i j, i d, t}, \quad \forall t, \forall i, j, i d \in \Omega_{b} \\
z c_{g, t-\text { period }}=z_{g, t}, \quad \forall t, \forall g \in \Omega_{g}
\end{gathered}
$$

Eqs. (40) and (41) are inequality constraints for voltage magnitude at each node and the phase angle differences between bus $i$ and $j$. Eqs. (42) and (43) explain numerical values of construction variables which are greater than or equal to a previous time at a specific time. Eqs. (44) and (45) represent a relationship between the two binary variables to consider algorithm with construction period. In Eq. (44) and (45), subscript period means construction period.

\section{Simulation Results}

\subsection{Application to the Garver's 6-bus system}

The proposed method has been applied to the Garver's 6-bus system shown in Fig. 4 [28, 29]. It is one of the most frequently used small scale sample power systems for the transmission expansion planning study. As shown in the figure, it is assumed that two generators on bus 1 and 3, and six transmission lines are already existed in the system. Bus 6 is also already decided to be built with fixed schedule.

Based on a simple topological analysis, it can be assumed that, without loss of generality, the transmission lines between the adjacent buses such as 1-2 and 1-4 have better economics than the transmission lines 1-3 and 1-6.

The load data are given in Table 1 . The real power

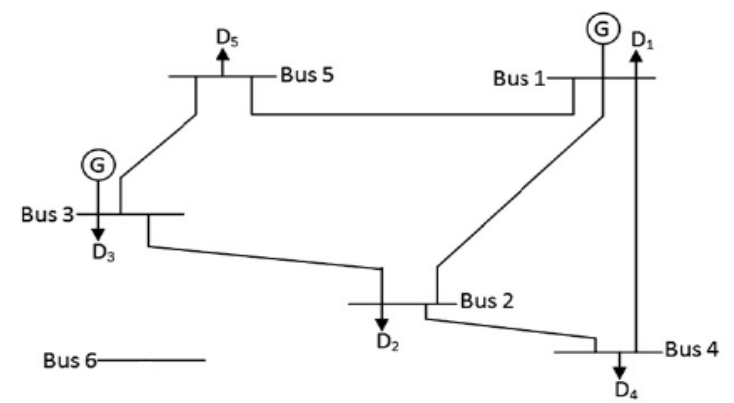

Fig. 4. Garver's 6-bus system

Table 1. Data for electricity demand [28]

\begin{tabular}{c|c|c}
\hline Bus No. & PD (MW) & QD (MVar) \\
\hline 1 & 80 & 11 \\
2 & 98 & 33 \\
3 & 85 & 6 \\
4 & 92 & 22 \\
5 & 77 & 33 \\
6 & 67 & 20 \\
\hline
\end{tabular}


demand of each bus is assumed to increase by $8 \%, 10 \%$, $20 \%$ and $25 \%$ from the previous year. The reactive demand of each bus is assumed to increase by $10 \%$ every year during the planning period. The data for the generators (both existing ones and new candidates) and the transmission lines are given in Table 2 and 3, respectively. All the voltage deviation limits are set to $\pm 5 \%$ of the nominal values. The voltage angles between adjacent buses are limited at $\pm 35^{\circ}$ or $\pm 0.61 \mathrm{rad}$, which is typical values for the practical transmission line loadability [30].

The proposed method is implemented using the MINOS and BARON solvers which are among typical NLP and MIP solvers available with GAMS (General Algebraic Modeling System) (http://www.gams.com) [31].

As shown in the Table 4 and 5, both methods come to the same results except that the proposed GBD method is almost twice faster than the combinatorial search method. As shown in the Table 5, it takes about 50 minutes for the combinatorial search method to solve the integrated optimization problem, whereas the proposed GBD method

Table 2. Data for the existing and candidate generators

\begin{tabular}{c|c|c|c|c}
\hline Gen No. & 1 & 2 & 3 & 4 \\
\hline $\mathrm{S}_{\max }(\mathrm{MVA})$ & 300 & 390 & 642 & 400 \\
\hline Location at Bus & 1 & 3 & 6 & 5 \\
\hline$a_{g}\left(\$ / \mathrm{MW}^{2}\right)$ & 0.01 & 0.015 & 0.005 & 0.002 \\
\hline$b_{g}(\$ / \mathrm{MW})$ & 5 & 7 & 8.5 & 10 \\
\hline Investment cost(M\$) & $\cdot$ & $\cdot$ & 1000 & 2000 \\
\hline
\end{tabular}

Table 3. Data for the candidate transmission lines

\begin{tabular}{c|c|c|c|c}
\hline Corridor & $\begin{array}{c}\mathrm{x}_{\mathrm{ij}} \\
\text { (p.u) }\end{array}$ & $\begin{array}{c}\text { Capacity } \\
\text { (MVA) }\end{array}$ & $\begin{array}{c}\text { Cost } \\
(\mathrm{M} \$)\end{array}$ & $\begin{array}{c}\text { Construction } \\
\text { period }\end{array}$ \\
\hline $1-2$ & 0.4 & 100 & 40 & 1 \\
\hline $1-4$ & 0.6 & 80 & 60 & 1 \\
\hline $1-5$ & 0.2 & 100 & 22 & 1 \\
\hline $2-3$ & 0.2 & 100 & 24 & 1 \\
\hline $2-4$ & 0.4 & 100 & 41 & 1 \\
\hline $2-5$ & 0.31 & 100 & 31 & 1 \\
\hline $2-6$ & 0.31 & 100 & 30 & 1 \\
\hline $3-5$ & 0.2 & 100 & 20 & 1 \\
\hline $3-6$ & 0.48 & 100 & 48 & 1 \\
\hline $4-6$ & 0.3 & 100 & 33 & 1 \\
\hline
\end{tabular}

Table 4. Generation expansion planning results

\begin{tabular}{c|c|c}
\hline \multirow{2}{*}{$\begin{array}{c}\text { Expansion } \\
\text { method }\end{array}$} & \multicolumn{2}{|c}{ Generator expansion result } \\
\cline { 2 - 3 } & $\begin{array}{c}\text { Combinatorial search } \\
\text { (Branch and cut method) }\end{array}$ & $\begin{array}{c}\text { Proposed } \\
\text { GBD method }\end{array}$ \\
\hline Year 1 & G3 & G3 \\
\hline
\end{tabular}

Table 5. Transmission line expansion planning results

\begin{tabular}{c|c|c}
\hline \multirow{2}{*}{$\begin{array}{c}\text { Expansion } \\
\text { method }\end{array}$} & \multicolumn{2}{|c}{ Transmission line expansion result } \\
\cline { 2 - 3 } & $\begin{array}{c}\text { Combinatorial search } \\
\text { (Branch and cut method) }\end{array}$ & $\begin{array}{c}\text { Proposed } \\
\text { GBD method }\end{array}$ \\
\hline Year 1 & $(2-6)$ & $(2-6)$ \\
\hline Year 2 & $(4-6)$ & $(4-6)$ \\
\hline Year 4 & $(1-4),(3-5),(3-6)$ & $(1-4),(3-5),(3-6)$ \\
\hline Computation time & $50 \mathrm{~m}$ & $32 \mathrm{~m}$ \\
\hline
\end{tabular}

takes only about 30 minutes. It is obvious that computation time of the combinatorial search method would be exponentially increased as the size of the studied system becomes larger.

The optimization result of the proposed method is shown in Table 4, Table 5 and Fig. 5. For comparison, the optimization result obtained from the combinatorial search method is also included. The combinatorial search method used here is the branch and cut method [18].

As shown in Fig. 6 and Table 6, the total cost of the proposed method with AC power flow model is higher than that of the method with DC power flow model, which can be somewhat misleading that the method based on DC power flow is superior to the proposed method based on AC power flow model. This is mainly because the solution space (feasible region) of the problem based on the DC power flow model is wider than that of the problem based on the AC power flow model, while the extra solution space of DC power flow model is mostly infeasible from AC point of view (voltage limit or reactive power constraint violation). Therefore, it is natural that the total

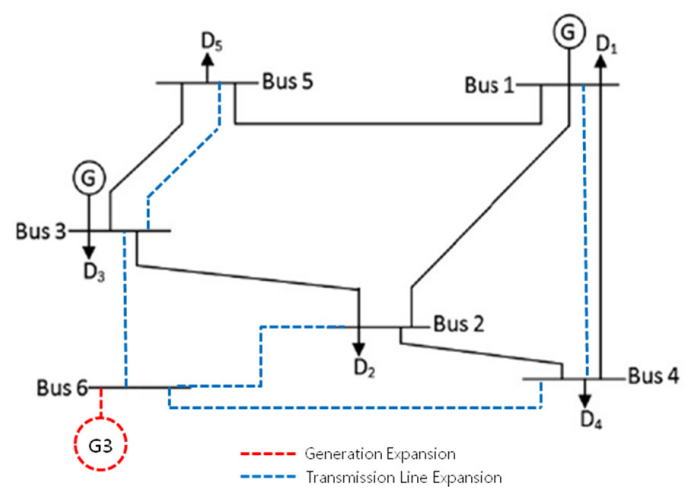

Fig. 5. Expansion results of the proposed method

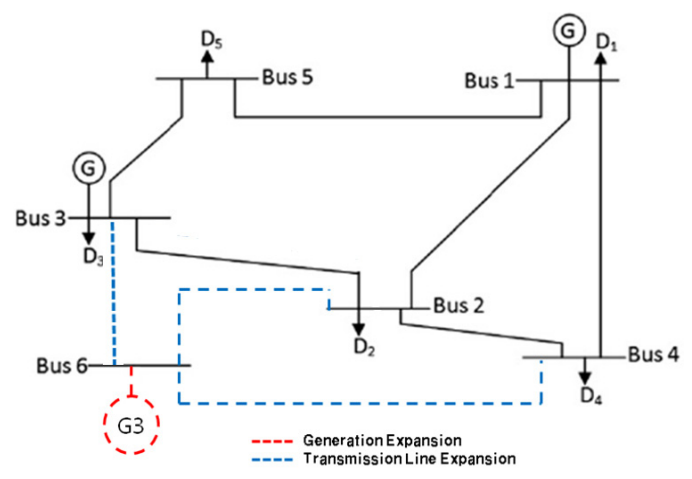

Fig. 6. Expansion results of the GBD method with DC power flow model

Table 6. Comparisons of the proposed method (linearized AC power flow) and the DC power flow model

\begin{tabular}{c|c|c}
\hline & Proposed method & DC power flow model \\
\hline $\begin{array}{c}\text { Expansion Result } \\
\text { (last year) }\end{array}$ & $\begin{array}{c}\text { G3, (2-6), (4-6), } \\
(1-4),(3-5),(3-6)\end{array}$ & G3, (2-6), (3-6), (4-6) \\
\hline Objective value & 25706.01 & 25542.61 \\
\hline
\end{tabular}


Table 7. Comparisons of GEP, TEP and IGTEP

\begin{tabular}{c|c|c}
\hline & $\begin{array}{c}\text { Expansion result } \\
\text { (last year) }\end{array}$ & $\begin{array}{c}\text { Objective } \\
\text { value }\end{array}$ \\
\hline $\begin{array}{c}\text { High transmission } \\
\text { cost }\end{array}$ & $\mathrm{G} 3$ (Year 1), G4(Year 3) & 27198.86 \\
\hline $\begin{array}{c}\text { High generation } \\
\text { cost }\end{array}$ & $(1-4),(1-5),(2-3),(2-6),(3-6)$ & 27172.58 \\
\hline IGTEP & $\mathrm{G} 3,(2-6),(4-6),(1-4),(3-5),(3-6)$ & 25706.01 \\
\hline
\end{tabular}

Table 8. Calculation of voltage magnitude and phase angle (the results of the final year)

\begin{tabular}{c|c|c|c|c}
\hline \multirow{2}{*}{$\begin{array}{c}\text { Bus } \\
\text { No. }\end{array}$} & \multicolumn{2}{|c|}{$\begin{array}{c}\text { Proposed } \\
\text { GBD method }\end{array}$} & \multicolumn{2}{c}{$\begin{array}{c}\text { Full AC power flow } \\
\text { model (PSS/e) }\end{array}$} \\
\cline { 2 - 5 } & $V_{i}$ & $\theta_{i}$ & $V_{i}$ & $\theta_{i}$ \\
\hline 1 & 1 & 0 & 1 & 0 \\
\hline 2 & 1 & -0.046 & 0.986 & -0.046 \\
\hline 3 & 1.043 & -0.008 & 1.03 & -0.008 \\
\hline 4 & 0.984 & -0.057 & 0.971 & -0.057 \\
\hline 5 & 1.02 & -0.035 & 1.011 & -0.035 \\
\hline 6 & 0.984 & 0.047 & 0.967 & 0.049 \\
\hline
\end{tabular}

cost of the optimal solution with DC power flow model is lower than that of the optimal solution with $\mathrm{AC}$ power flow model.

The optimal solution of the IGTEP severely depends on the relative costs (mostly capital costs) between generators and transmission lines. Two extreme cases are studied: when the transmission cost is relatively higher than generation cost (generation-only expansion planning) and when the generation cost is relatively higher than transmission cost (transmission-only expansion planning). The results are summarized in Table 7, where we can figure out that the integrated optimization is in between those two extreme cases. IGTEP requires much longer period to get the optimal solution compared to the other extreme cases, but the result can be superior to other extreme cases.

Table 8 shows the voltage magnitudes and phase angles which are by-product of the proposed method and they are compared with the simulation results from PSS/e, one of the most frequently used AC power flow model. As can be seen in the table, voltage magnitudes and phase angles from the proposed method are not very different from the result of the commercial AC power flow model.

\subsection{Application to the IEEE 30-bus system [32]}

In addition to the Garver's 6-bus system, the IEEE 30bus system has been studied in order to verify the performance of the proposed method when applied to a larger scale power system.

The planning horizon is set to 10 years and the demand is assumed to increase by $2.5 \%$ every year. The generator and network parameters are chosen based on authors' own discretion, which are described in Appendix. In order to reduce the overall simulation time, the maximum number of generators and transmission lines that can be built in a

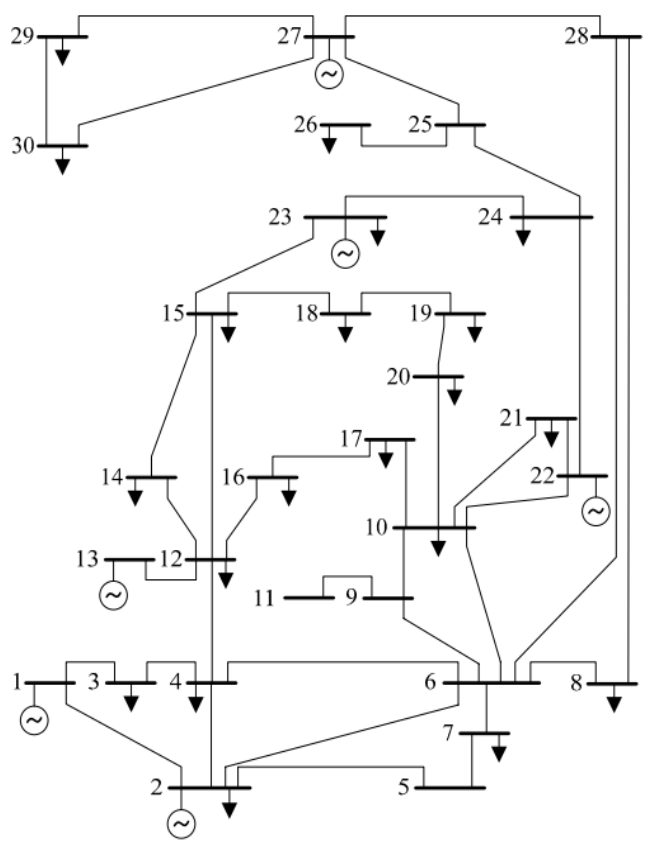

Fig. 7. The IEEE 30-bus test system [32]

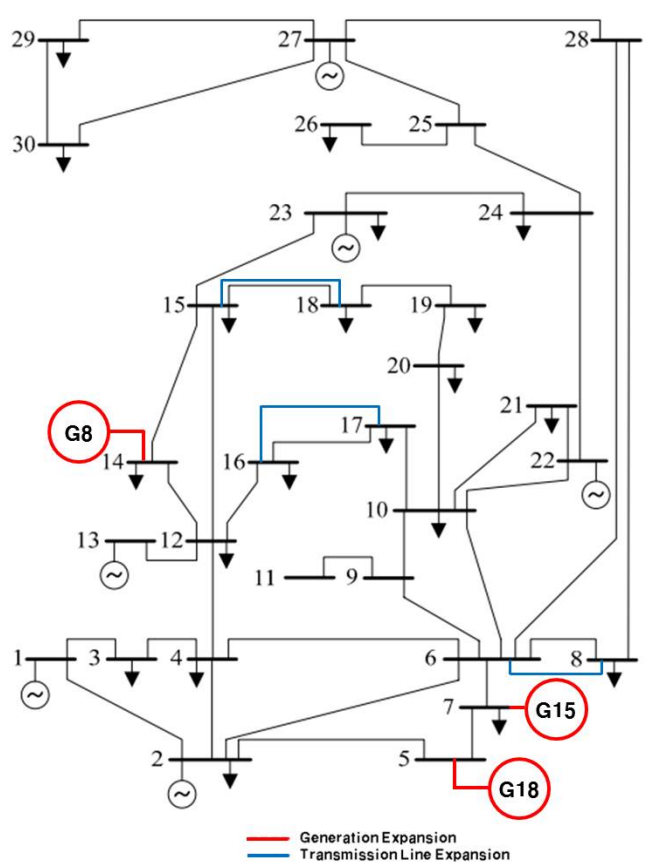

Fig. 8. Expansion results of the proposed method

Table 9. Generation expansion planning results

\begin{tabular}{c|c|c}
\hline \multirow{2}{*}{$\begin{array}{c}\text { Expansion } \\
\text { method }\end{array}$} & \multicolumn{2}{|c}{ Generator expansion result } \\
\cline { 2 - 3 } & $\begin{array}{c}\text { Combinatorial search } \\
\text { (Branch and cut method) }\end{array}$ & $\begin{array}{c}\text { Proposed } \\
\text { GBD method }\end{array}$ \\
\hline Year 4 & - & G15 \\
\hline Year 9 & - & G18 \\
\hline Year 10 & - & G8 \\
\hline
\end{tabular}

year, or Eq. (36) and (37), are set to one, respectively.

The simulation results are shown in Table 9, Table 10 
Table 10. Transmission line expansion planning results

\begin{tabular}{c|c|c}
\hline \multirow{2}{*}{$\begin{array}{c}\text { Expansion } \\
\text { method }\end{array}$} & \multicolumn{2}{|c}{ Transmission line expansion result } \\
\cline { 2 - 3 } & $\begin{array}{c}\text { Combinatorial search } \\
\text { (Branch and cut method) }\end{array}$ & $\begin{array}{c}\text { Proposed } \\
\text { GBD method }\end{array}$ \\
\hline Year 8 & - & $(15-18)$ \\
\hline Year 9 & - & $(6-8)$ \\
\hline Year 10 & - & $(16-17)$ \\
\hline Computation time & - & $1 \mathrm{~h} \mathrm{30m}$ \\
\hline
\end{tabular}

and Fig. 8. As shown in the Table 10, the proposed method can obtain the solution in a reasonable period, but the combinatorial method fails to converge to the reasonable result even after 72 hours.

We may carefully assert that the proposed method is less sensitive to the size of the problem than the combinatorial search method although a rigorous mathematical justification is requested.

\section{Conclusions}

In order to find the optimal solution of the IGTEP problem, the combinatorial search method and the GBD method have been used extensively. But, most of the existing researches are based on the DC power flow model. It is natural desire for the power system planner to use the AC power flow model in the IGTEP problem in order to deal with various constraints related to bus voltage and reactive power.

Though the IGTEP problem with DC power flow model has a very structure that the GBD method can easily be applied, the extension of the method to the AC power flow model is hindered due to severe nonlinearity and nonconvexity of the AC power flow equation. So, this paper proposed the GBD method based on the linearized version of the AC power flow model. The main idea of this paper is that the linearization is performed in a limited way in order not to deteriorate the accuracy of the result.

The proposed method was successfully applied to the Garver's 6-bus system and the IEEE 30-bus system which are frequently used for transmission expansion planning studies. However, further mathematical and simulation researches are still needed to improve the performance of the proposed method to be applied to the real scale power system.

\section{Acknowledgements}

This work was supported by the Human Resources Program in Energy Technology of the Korea Institute of Energy Technology Evaluation and Planning (KETEP), granted financial resource from the Ministry of Trade, Industry \& Energy, Republic of Korea (No.2015
4030200670), and a 2-Year Research Grant of Pusan National University

\section{Appendix}

Table A-1. Generators data (existing and candidates) [32]

\begin{tabular}{c|c|c|c|c|c}
\hline $\begin{array}{c}\text { Gen } \\
\text { No. }\end{array}$ & $\begin{array}{c}\mathrm{S}_{\max } \\
(\mathrm{MVA})\end{array}$ & $\begin{array}{c}\text { Location at } \\
\text { Bus }\end{array}$ & $\begin{array}{c}a_{g} \\
\left(\$ / \mathrm{MW}^{2}\right)\end{array}$ & $\begin{array}{c}b_{g} \\
(\$ / \mathrm{MW})\end{array}$ & $\begin{array}{c}\text { Investment } \\
\text { cost(M\$) }\end{array}$ \\
\hline 1 & 80 & 1 & 0.02 & 15 & - \\
\hline 2 & 80 & 2 & 0.0175 & 14.75 & - \\
\hline 3 & 50 & 13 & 0.025 & 16 & - \\
\hline 4 & 50 & 22 & 0.0625 & 14 & - \\
\hline 5 & 30 & 23 & 0.025 & 16 & - \\
\hline 6 & 55 & 27 & 0.0083 & 15.25 & - \\
\hline 7 & 80 & 3 & 0.02 & 15 & 59645 \\
\hline 8 & 80 & 14 & 0.0175 & 14.75 & 37810 \\
\hline 9 & 50 & 5 & 0.025 & 16 & 44650 \\
\hline 10 & 50 & 13 & 0.0625 & 14 & 87630 \\
\hline 11 & 30 & 7 & 0.025 & 16 & 50990 \\
\hline 12 & 55 & 14 & 0.0083 & 15.25 & 59580 \\
\hline 13 & 80 & 8 & 0.02 & 15 & 84530 \\
\hline 14 & 80 & 19 & 0.0175 & 14.75 & 38870 \\
\hline 15 & 50 & 7 & 0.025 & 16 & 23030 \\
\hline 16 & 50 & 24 & 0.0625 & 14 & 98300 \\
\hline 17 & 30 & 11 & 0.025 & 16 & 88560 \\
\hline 18 & 55 & 5 & 0.0083 & 15.25 & 24340 \\
\hline 19 & 80 & 16 & 0.02 & 15 & 60210 \\
\hline 20 & 80 & 20 & 0.0175 & 14.75 & 53710 \\
\hline & & & & & \\
\hline
\end{tabular}

Table A-2. Transmission lines data (candidates) [32]

\begin{tabular}{|c|c|c|c|}
\hline Corridor & $\mathrm{x}_{\mathrm{ij}}$ (p.u) & Capacity(MVA) & $\operatorname{Cost}(\mathrm{M} \$)$ \\
\hline $1-2$ & 0.06 & 65 & 3326 \\
\hline $1-3$ & 0.19 & 65 & 9620 \\
\hline $2-4$ & 0.17 & 32.5 & 2980 \\
\hline $3-4$ & 0.04 & 65 & 2740 \\
\hline $2-5$ & 0.2 & 65 & 5200 \\
\hline $2-6$ & 0.18 & 32.5 & 4730 \\
\hline $4-6$ & 0.04 & 45 & 2809 \\
\hline $5-7$ & 0.12 & 35 & 3743 \\
\hline $6-7$ & 0.08 & 65 & 2926 \\
\hline $6-8$ & 0.04 & 16 & 1184 \\
\hline $6-9$ & 0.21 & 32.5 & 2928 \\
\hline $6-10$ & 0.56 & 16 & 1271 \\
\hline $9-11$ & 0.21 & 32.5 & 3072 \\
\hline $9-10$ & 0.11 & 32.5 & 2290 \\
\hline $4-12$ & 0.26 & 32.5 & 4384 \\
\hline $12-13$ & 0.14 & 32.5 & 1513 \\
\hline $12-14$ & 0.26 & 16 & 3943 \\
\hline $12-15$ & 0.13 & 16 & 3360 \\
\hline $12-16$ & 0.2 & 16 & 1214 \\
\hline $14-15$ & 0.2 & 8 & 1914 \\
\hline $16-17$ & 0.19 & 8 & 984 \\
\hline $15-18$ & 0.22 & 8 & 656 \\
\hline $18-19$ & 0.13 & 8 & 4554 \\
\hline $19-20$ & 0.07 & 16 & 3154 \\
\hline $10-20$ & 0.21 & 16 & 1868 \\
\hline $10-17$ & 0.08 & 16 & 1483 \\
\hline $10-21$ & 0.07 & 16 & 2187 \\
\hline $10-22$ & 0.15 & 16 & 3897 \\
\hline $21-22$ & 0.02 & 16 & 4384 \\
\hline $15-23$ & 0.2 & 8 & 3326 \\
\hline $22-24$ & 0.18 & 8 & 2734 \\
\hline $23-24$ & 0.27 & 8 & 4552 \\
\hline $24-25$ & 0.33 & 8 & 8911 \\
\hline
\end{tabular}




\begin{tabular}{c|c|c|c}
\hline $25-26$ & 0.38 & 8 & 2710 \\
\hline $25-27$ & 0.21 & 8 & 1436 \\
\hline $28-27$ & 0.4 & 32.5 & 3906 \\
\hline $27-29$ & 0.42 & 8 & 2408 \\
\hline $27-30$ & 0.6 & 8 & 3550 \\
\hline $29-30$ & 0.45 & 8 & 4643 \\
\hline $8-28$ & 0.2 & 16 & 4443 \\
\hline $6-28$ & 0.06 & 16 & 2880 \\
\hline
\end{tabular}

Table A-3. Data for electricity demand [32]

\begin{tabular}{|c|c|c|}
\hline Bus No. & PD (MW) & QD (MVar) \\
\hline 2 & 21.7 & 7 \\
\hline 3 & 2.4 & 1 \\
\hline 4 & 67.6 & 23 \\
\hline 5 & 34.2 & 11 \\
\hline 7 & 22.8 & 8 \\
\hline 8 & 30 & 10 \\
\hline 10 & 5.8 & 2 \\
\hline 12 & 11.2 & 4 \\
\hline 14 & 6.2 & 2 \\
\hline 15 & 8.2 & 3 \\
\hline 16 & 3.5 & 1 \\
\hline 17 & 9 & 3 \\
\hline 18 & 3.2 & 1 \\
\hline 19 & 9.5 & 3 \\
\hline 20 & 2.2 & 1 \\
\hline 21 & 17.5 & 6 \\
\hline 23 & 3.2 & 1 \\
\hline 24 & 8.7 & 3 \\
\hline 26 & 3.5 & 1 \\
\hline 29 & 2.4 & 1 \\
\hline 30 & 10.6 & 4 \\
\hline
\end{tabular}

\section{References}

[1] K. Ramachandran and J.D. Sharma, "A Method for Generation and Transmission Planning," Computers and Electrical Engineering, Vol. 5, pp. 171-178, 1978

[2] M.V.F. Pereira, M.V.G. Pinto, S.H.F. Cunha and G.C. Oliveira, "A Decomposition Approach to Automated Generation/Transmission Expansion Planning," IEEE Transactions on Power Apparatus and Systems, Vol. PAS-104, No. 11, pp. 3074-3083, 1985

[3] L. Wenyuan and R. Billinton, "A Minimum Cost Assessment Method for Composite Generation and Transmission System Expansion Planning," IEEE Transactions on Power Systems, Vol. 8, No. 2, pp. 628-635, 1993

[4] M.L. Baughman, S.N. Siddiqi and J.W. Zarnikau, "Integrating Transmission into IRP Part 1: Analytical Approach", IEEE transactions on Power Systems, Vol. 10, No. 3, pp. 1652-1659, 1995

[5] M.L. Baughman, S.N. Siddiqi and J.W. Zarnikau, "Integrating Transmission into IRP Part 2: Case Study", IEEE Transactions on Power Systems, Vol. 10, No. 3, pp. 1652-1659, 1995

[6] H. M. D. R. H. Samarakoon, R. M. Shrestha, O. Fujiwara, "A Mixed Integer Programming Model for
Transmission Expansion Planning with Generation Location Selection", International Journal of Electrical Power and Energy Systems, Vol. 23, No. 4, pp. 285-293, May. 2001

[7] S.-M. Han, K.-H. Chung and B.H. Kim, "Integrated Power System Planning considering Generation \& Network Planning," in Proc. of 2005 KIEE Summer Meeting, pp. 18-20, 2005

[8] J. Alvarez Lopez, K. Ponnambalam and V.H. Quintana, "Generation and Transmission Expansion under Risk Using Stochastic Programming", IEEE Transactions on Power Systems, Vol. 22, No. 3, Aug. 2007

[9] J.H. Roh, M. Shahidehpour and Lei Wu, "MarketBased Coordination of Transmission and Generation Capacity Planning", IEEE Transactions on Power Systems, Vol. 22, No. 4, pp. 1406-1419, Nov. 2007

[10] J.H. Roh, M. Shahidehpour and Lei Wu, "MarketBased Generation and Transmission Planning with Uncertainties", IEEE Transactions on Power Systems, Vol. 24, No. 3, pp. 1587-1598, Aug. 2009

[11] M.S. Sepasian, H. Seifi, A.A. Foroud and A.R. Hatame, "A Multiyear Security Constrained Hybrid Generation-Transmission Expansion Planning Algorithm Including Fuel Supply Costs", IEEE Transactions on Power Systems, Vol. 24, No. 3, pp. 1609-1618, Aug. 2009

[12] N. Hariyanto, M. Nurdin, Y. Haroen and C. Machbub, "Decentralized and Simultaneous Generation and Transmission Expansion Planning through Cooperative Game Theory', Int. J. Electr. Eng. Inf, Vol. 1, No. 2, pp. 149-164, 2009

[13] A. Motamedi, H. Zareipour, M.O. Buygi and W.D. Rosehart, "A Transmission Planning Framework Considering Future Generation Expansions in Electricity Markets", IEEE Transactions on Power Systems, Vol. 25, No. 4, pp. 1987-1995, Nov. 2010

[14] B. Alizadeh and S. Jadid, "Reliability Constrained Coordination of Generation and Transmission Expansion Planning in Power Systems using Mixed Integer Programming", IET Generation, Transmission and Distribution., Vol. 5, No. 9, pp. 948-960. May. 2011

[15] I. Sharan and R. Balasubramanian, "Integrated Generation and Transmission Expansion Planning Includ-ing Power and Fuel Transportation Constraints," Energy Policy, Vol. 43, No. 1, pp. 275-284, Apr. 2012

[16] R.-A. Hooshmand, R. Hemmati and M. Parastegari, "Combination of AC Transmission Expansion Planning and Reactive Power Planning in the Restructured Power System," Energy Conversion and Management, Vol. 55, pp. 26-35, 2012

[17] F. Barati, A. Nateghi, H. Seifi and M.S. Sepasian, "Generation and Transmission expansion planning with considering natural gas network", Electrical Engineering on $201321^{\text {st }}$ Iranian Conference, pp. 1-7, May. 2013 
[18] H.T. Kim and W. Kim, "Integrated Optimization of Combined Generation and Transmission Expansion Planning Considering Bus Voltage Limits", Journal of Electrical Engineering \& Technology, Vol. 9, No. 4, pp. 1202-1209, Sep. 2014

[19] H.G. Stoll, "Least-Cost Electric Utility Planning", John Wiley \& Sons, 1989

[20] A. Floudas, Nonlinear and Mixed-Integer Optimization, New York:Oxford University Press, 1995

[21] S. Binato, M.V.F. Pereira and S. Granvile, "A new Benders Decomposition Approach to Solve Power Transmission Network Design Problems", IEEE Transactions on Power Systems, Vol. 16, No. 2, pp. 235-240, May. 2001

[22] S. Lumbreras and A. Ramos, "Transmission Expansion Planning Using an Efficient Version of Bender's Decomposition:A Case Study", 2013 IEEE Grenoble Power Tech, pp. 1-7, 2013

[23] S. Dehghan, H. Saboori, A. Kazemi and S. Jadid, "Transmission Network Expansion Planning Using a DEA-based Bender's Decomposition", $201018^{\text {th }}$ Iranian Conference on Electrical Engineering, pp. 955-960, 2010

[24] V.A. Levi and M.S. Calovic, "Linear-Programmingbased Decomposition Method for Optimal Planning of Transmission Network Investments", IEE Proceedings $\mathrm{C}$ on Generation, Transmission and Distribution, Vol. 140, No. 6, pp. 516-522, 1993

[25] S. Asadamongkol and B. Eua-arporn, "Transmission Expansion Planning with AC model based on Generalized Benders Decomposition", International Journal of Electrical Power and Energy Systems, Vol. 47, pp. 402-407, May. 2013

[26] A. M. Geoffrion, "Generalized Benders Decomposition", Journal of Optimization Theory and Applications, Vol. 10, No. 4, 1972

[27] J.J. Grainger and W.D. Stevenson, JR., Power System Analysis, McGraw-Hill, 1994

[28] H. Zhang, G.T. Heydt, V. Vijay and J. Quintero, “An Improved Network Model for Transmission Expansion Planning Considering Reactive Power and Network Losses", IEEE Transactions on Power Systems, pp. 3471-3479, Vol. 28, No. 3, Aug. 2013.

[29] L. L. Garver, "Transmission Network Estimation Using Linear Programming," IEEE Trans. on Power Apparatus and System, Vol. PAS-89, No. 7, pp. 16881697, Sep/Oct., 1970.

[30] J. D. Glover, M.S. Sarma and T.J. Overbye, Power System Analysis and Design, Cengage Learning, 2012

[31] R.E. Rosenthal, GAMS - A User's Guide, GAMS Development Corporation, 2012

[32] M. Shahidehpour, Y. Wang, Communication and Control in Electric Power Systems: Applications of Parallel and Distributed Processing, Wiley-IEEE, Jun. 2003

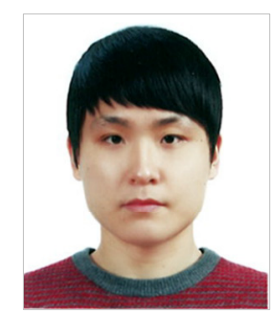

Hyoungtae Kim received B.S. and M.S degrees in Electronic and Electrical engineering from Pusan National University, Korea, in 2013 and 2015, respectively. Currently, he is pursuing his Ph.D. degree in Pusan National University. His research interests are power system economics and smart grid.

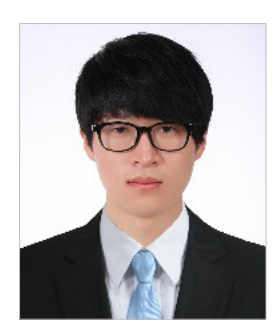

Sungwoo Lee received B.S. and M.S. degrees in Electronic and Electrical engineering from Pusan National University, Korea, in 2013 and 2015, respectively. Currently, he is pursuing his Ph.D. degree in Pusan National University. His research interests are power system economics and smart grid.

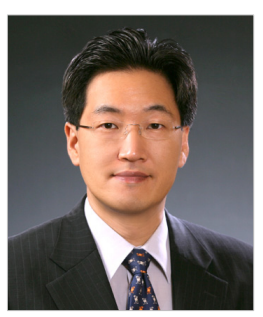

Wook Kim received B.S, M.S. and Ph.D. degrees in Electrical Engineering from Seoul National University in 1990, 1992 and 1997, respectively. For 1997-2011, he was with LG Industrial Systems, Samsung Securities and Korea Southern Power Co. Since Sep. 2011, he has been with Electrical Engineering Department at Pusan National University. He is also a research member of the Research Institute of Computer Information and Communication and LG Electronics Smart Control Center in Korea. His research interests include power economics, electricity and carbon trading, smart grid and optimization theory. 